\title{
Developments in Sheet Hydroforming for Complex Industrial Parts
}

\author{
M. Bakhshi-Jooybari, A. Gorji and M. Elyasi \\ Additional information is available at the end of the chapter
}

http://dx.doi.org/10.5772/48142

\section{Introduction}

Sheet-metal forming processes are technologically among the most important metalworking processes. Products made by these processes include a large variety of shapes and sizes. Typical examples are automobile bodies, aircraft panels, appliance bodies, kitchen utensils and beverage cans [1].

Among the various sheet-metal forming processes, hydroforming is one of the nontraditional ones. This process is also called hydromechanical forming, hydraulic forming or hydropunch forming. In hydroforming process, liquid is used as the medium of energy transfer to form the workpiece. The part is formed on a female die, with the liquid under pressure acting in place of a conventional solid punch [1].

Hydroforming is applied more and more in the modern manufacturing industry. Comparing with the solid punch stretching process, hydroforming process results in a better strain state in the workpiece, so that a deeper draw can be achieved. The friction between tools and blank is greatly reduced. The advantages of hydroforming include low tooling cost, flexibility and ease of operation, low tool wear, no damage to the surface of the sheet, and capability to form complex shapes [2].

Types of hydroforming process

Hydroforming process is divided into two main groups; sheet hydroforming and tube hydroforming. These are briefly stated in the following sections.

\section{Tube hydroforming process}

Tube HydroForming (THF) is a process of forming hollow parts with different cross sections by applying simultaneously an internal hydraulic pressure and axial compressive loads to force a tubular blank to conform to the shape of a given die. Geometry of die and workpiece, 
initial tube dimension, tube anisotropy, and internal pressure are of the important parameters in this process [3].

With the advancements in computer control and high-pressure hydraulic systems, this process has become a viable method for mass production, especially with the use of internal pressure of up to 6000 bars. Tube hydroforming offers several advantages as compared to conventional manufacturing processes. These advantages include; a) part consolidation, b) weight reduction through more efficient section design, c) improved structural strength and stiffness, d) lower tooling cost due to fewer parts, e) fewer secondary operations (no welding of sections required and holes may be pierced during hydroforming), and f) tight dimensional tolerances. Despite several benefits over stamping process, THF technology is still not fully implemented in the automotive industry due to its time-consuming part and process development [4].

In THF, compressive stresses occur in regions where the tube material is axially fed, and tensile stresses occur in expansion regions. The main failure modes are buckling, wrinkling (excessively high compressive stress) and bursting (excessively high tensile stress). It is clear that only an appropriate relationship between internal pressure curve versus time, and axial feed curve versus time, so called Loading Paths (LP), guarantees a successful THF process without any of the failures [5].

Hydroformed tubular parts vary over a wide range of shapes. This variety goes from a simple bulged tube to an engine cradle with multiple part features such as bends, protrusions, and complex cross sections. It is necessary to classify the THF parts into different categories with respect to common characteristics that they have in order to handle the design process more efficiently [6]. Figure 1 shows some types of parts which are produced in this process.

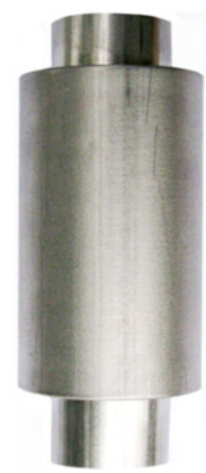

(a)

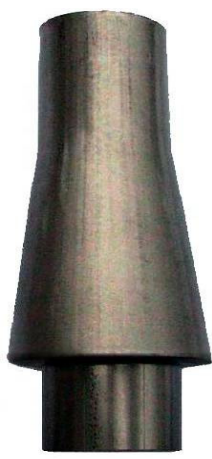

(b)

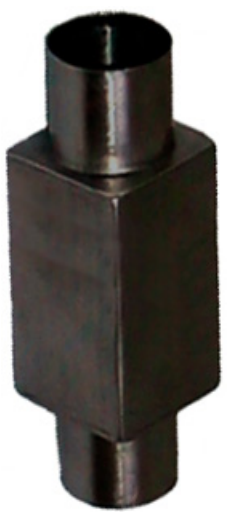

(c)

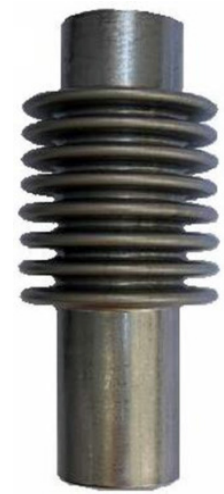

(d)

Figure 1. Tube hydroformed parts: (a) cylindrical stepped tube, (b) conical stepped tube, (c) rectangular stepped tube, (d) bellows [3-8] 


\section{Sheet hydroforming process}

Sheet hydroforming process is an alternative to drawing process where either punch or die is replaced by hydraulic medium, which generates the pressure and forms the part. Sheet hydroforming is classified into two types Sheet HydroForming with Punch (SHF-P) and Sheet HydroForming with Die (SHF-D). In SHF-P (Figure 2(a)), the hydraulic fluid is replaced with the die, while in SHF-D (Figure 2(b)), the hydraulic fluid is replaced with the punch. Absence of either punch or die in SHF process reduces the tooling cost [9].

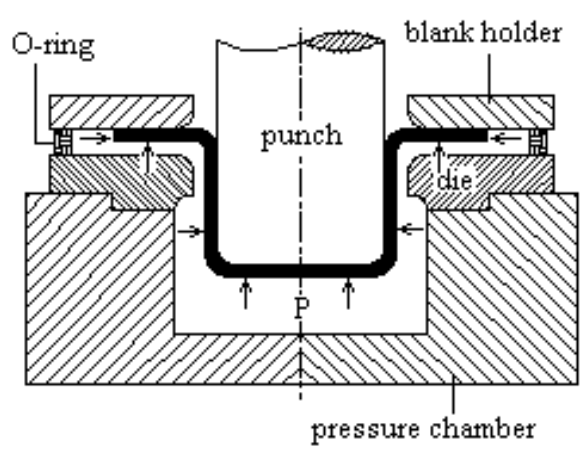

(a)

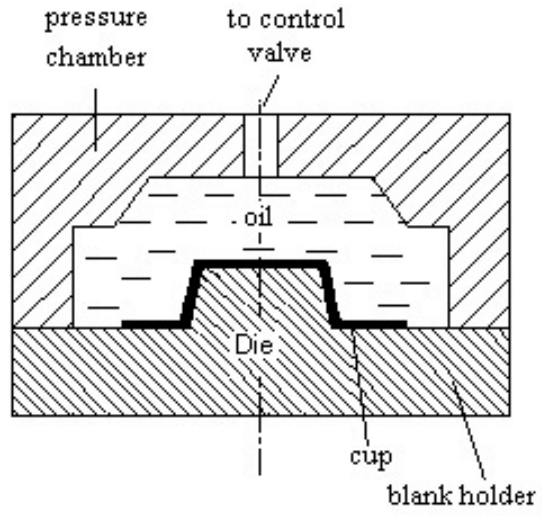

(b)

Figure 2. Schematic of the sheet hydroforming process: (a) SHF-P, (b) SHF-D [9]

Types of sheet hydroforming process

Some new sheet hydroforming processes have been introduced during the last few years. Thiruvarudchelvan and Lewis [10], Anwar Kandil [11], and Zhang and Danckert [12] studied the standard hydroforming process. Figure 3 illustrates the tool set-up for this process. An essential part of this set-up is a rubber diaphragm that seals the liquid in the pressure chamber. During the process, the flange of the part is kept pushed against the blank holder by the fluid pressure transmitted through the diaphragm. There are many advantages for the standard hydroforming process, such as better surface quality and forming of complex shapes. Meanwhile, it has some disadvantages, such as the requirement of heavy presses. In addition, it is easy to destroy the rubber diaphragm, since the diaphragm is nearly under a similar deformation as the workpiece [10]. The fluid pressure is very important in this process, because wrinkles will appear if the pressure is not sufficiently high. If the pressure is too high, the blank may be damaged by rupture [12].

The hydromechanical deep drawing process has been developed by researchers on the basis of the standard hydroforming technology [10, 12]. Figure 4(a) shows the schematic illustration of this process. The fluid pressure in this process can be produced by the downward movement of the punch, or be supplied by a hydraulic system, since no rubber diaphragm is used. The tool device in this process is similar to that in a conventional deep 
drawing. An O-ring is used to prevent the flow out of the fluid on the flange. By using this process, more local deformation, increased drawing ratio and forming of complicated parts are realized [10]. But, in this method the blank holder force is not sufficient to prevent complex shapes from wrinkling. In addition, the fluid pressure is very high that causes high clamping force, and thus, heavy presses are required.

The hydromechanical deep drawing process has been developed as the radial hydromechanical deep drawing (hydro-rim) process (Figure 4(b)) [13]. In this process, when the punch goes down into the die cavity, the blank is forced into the die cavity filled with liquid. The liquid will be pressurized and will push the blank tightly on the punch surface. Also, the liquid pressure exists around the blank rim. This, in turn, can realize some forced radial feeding which is difficult in the current sheet hydroforming processes. In this method, since there is no clamping force (with rigid or semi-rigid part), wrinkling will occur easily in the forming of complex shapes.

In another investigation, Groche and Metz [14, 15] used an active-elastic blank holder system for high-pressure forming. Figure 5 illustrates the simplified schematic of this system. In this method, an elastic blank holder with a circular groove was used under the blank. The active-elastic blank holder system showed improvements with respect to the material flow in the flange area and reduced sheet thinning in critical corner regions of the workpiece. In this method the die set-up is complicated and the forming pressure is high.

Among the sheet hydroforming processes, hydrodynamic deep drawing assisted by radial pressure (HDDRP) has been used to form complex shapes and has a good drawing ratio [16]. This process is shown in Figure 6.

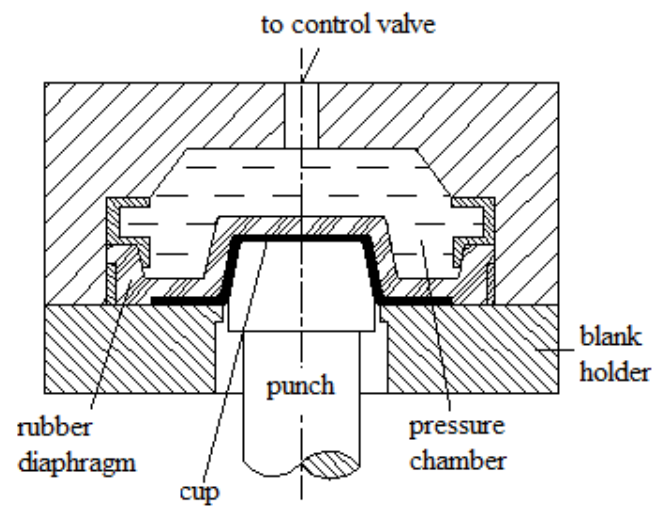

Figure 3. Tool set-up for standard hydroforming [9] 


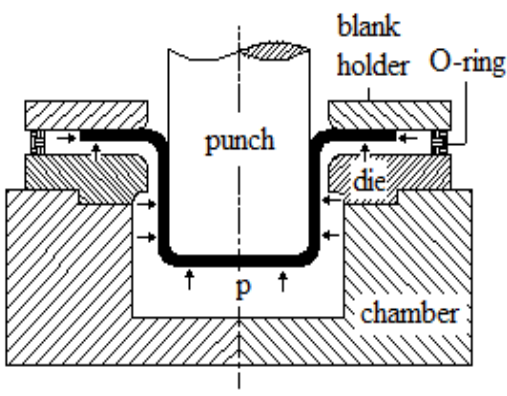

(a)

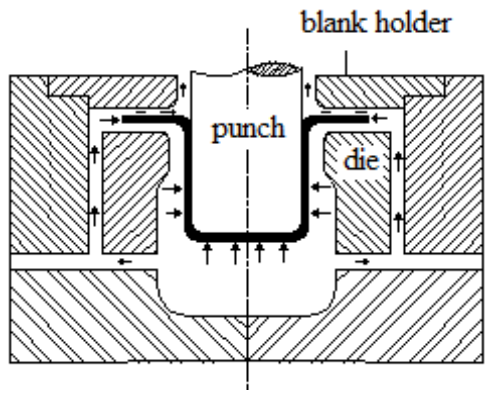

(b)

Figure 4. Tool set-up for: (a) hydromechanical, (b) hydro-rim, deep drawing process [9]

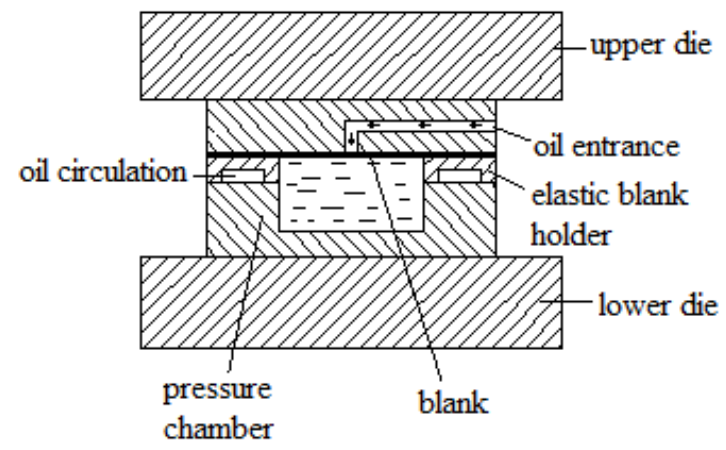

Figure 5. A simplified schematic of tool set-up for active-elastic blank holder system [9]

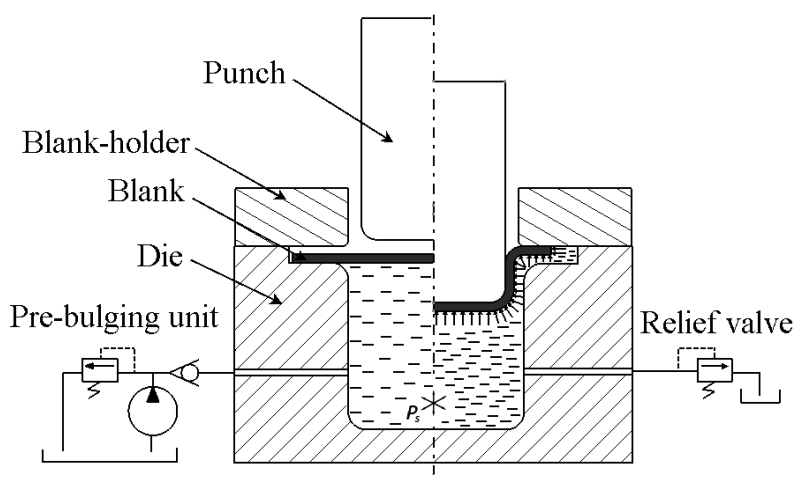

Figure 6. A simplified schematic of tool set-up for hydrodynamic deep drawing [16]

\section{Applications and limitations of sheet hydroforming process [9-16]}

Each of the sheet hydroforming mentioned above has its own limitations and applications. Sheet hydroforming process would be useful in reducing weight and cost simultaneously by 
improving structural integrity, strength and rigidity. In addition, this process satisfies these requirements with utilizing the common and available material efficiency.

Saving in tooling, material, design, production and assembly will altogether contribute reducing the overall cost of a sheet hydroforming part. Elimination or decrease of welds and welding operations is an additional of the overall cost.

A reduction in number of production steps and components in an assembly will be obtained with this process. This would reduce dimensional variations, and facilitate assembly operations.

Following is a list of potential advantages gained with the use of sheet hydroforming technology:

1. Reduction in weight

2. Increase in stiffness and rigidity

3. Economic material utilization

4. Complex shaped and various part types

5. Reduction in number of steps during manufacture and assembly (reduced welding and associated fixturing)

6. Reduction in overall cost per part or cost of assembly

7. Tight tolerances with good dimensional characteristics and less variation

8. Good surface finish

In contrast, longer process cycle and higher tool cost are limitations of this process.

\subsection{Selection of a sheet hydroforming process for a complex industrial workpiece}

The range of the applications of any sheet hydroforming process is limited. Not all the processes can be used for complex industrial parts. In this section, the criteria in selecting a specific process will be explained, such as the high drawing ratio, control of wrinkling, and ease of applying internal pressure.

\section{Case study: Introducing two complex industrial workpieces}

Case study I [9]

Figure 7 shows the photograph of the product that is used in lightning industry. The part is made of Al 1100 sheet with a thickness of $1 \mathrm{~mm}$ and with strain hardening equation of $\sigma=610$ $\varepsilon^{0.24}[\mathrm{MPa}]$. Currently, it is produced in the collaborating industry by the conventional deep drawing process in several stages.

Three main features of the workpiece are:

1. It has a very complex shape with special internal profile that should be produced with high precision and surface finish. 
2. There is a sharp edge on the workpiece that is specified in Figure 7(a). This is the most complex region of the workpiece to be formed.

3. The flange area of the workpiece that is shown in Figure 7(b), should be produced with good accuracy and with no wrinkle.

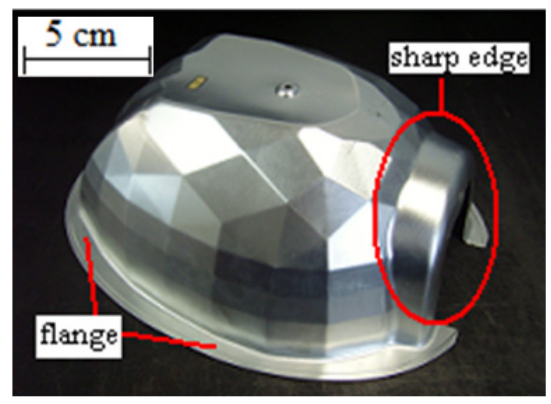

(a)

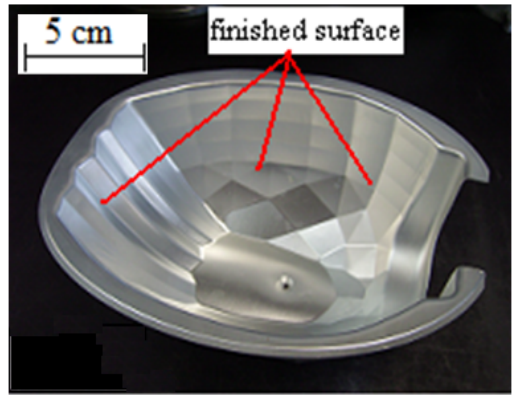

(b)

Figure 7. Case study 1; The part of lightning industry (after trimming and coating): (a) internal view, (b) external view

\section{Case study II [17]}

Drawing of conical parts is considerably more difficult than the deep drawing of cylindrical cups. Conical shape forming through conventional deep drawing is shown in Figure 8. As it is shown in the figure, due to small contact area of the punch tip with the blank at initial stages of deformation, high stresses are applied to this area of the sheet. This may cause bursting in the sheet. In addition, when forming conical cups through conventional deep drawing, wrinkling occurs on the sheet wall because the blank is free between the punch and the die $[18,19]$. Thus, conical parts are normally formed by multi-stage deep drawing [18], spinning [20] or explosive forming [21].

Because of the large radial tensile stresses, small drawing ratios must be used in each stage when making conical components in multi-stage deep drawing. In addition, the ratio of the sheet thickness to the initial blank diameter influences the limiting drawing ratio to a greater extent than when drawing cylindrical parts. The limiting drawing ratio also depends on the cone angle and the ratio of the largest to the smallest cone diameter [18].

\section{Experimental set-up}

In all the hydroforming processes a universal testing machine (Figure 9(a)) and a hydraulic unit (Figure 9(b)) are used. In addition, the hydraulic unit was used as the pressure medium to form the workpiece. A control valve with a maximum pressure of $50 \mathrm{MPa}$ regulated the liquid flow to maintain the required pressure.

\section{Tool set-up: Case study I}

Figure 10 illustrates the schematic illustration of the new tool set-up proposed for this part. Figure 11 shows the photograph of the punch used for the part. The manufactured die-set is 


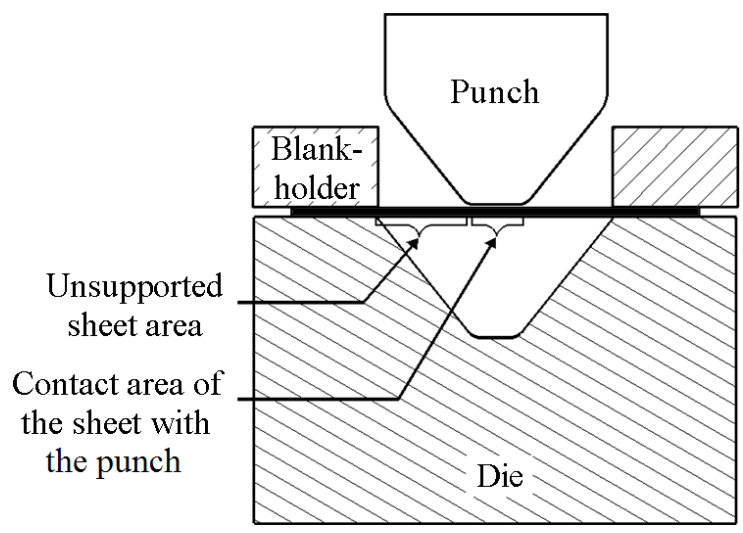

Figure 8. Schematic illustration of forming a conical part in conventional deep drawing

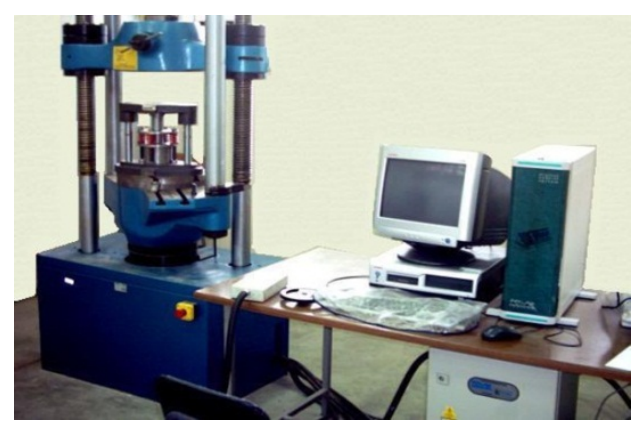

(a)

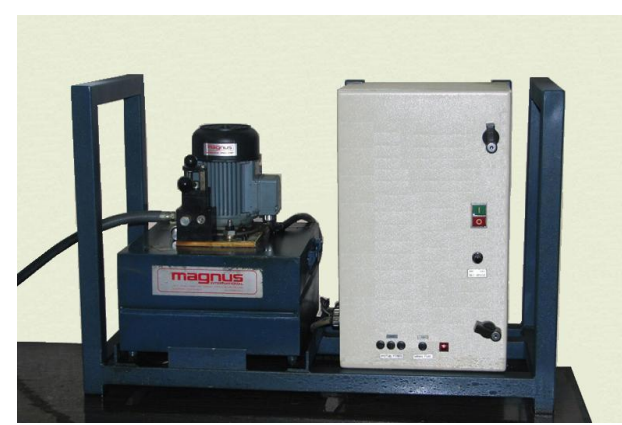

(b)

Figure 9. (a) Testing machine, (b) hydraulic unit

shown in Figure 12. It consists of a punch, a blank holder, a steel sheet ring, a die (oil container), a rubber diaphragm and an O-ring, which seals the liquid in the pressure chamber (die). As it is seen from the figure, the rubber diaphragm is only used in the region between the blank holder and die. Therefore, no diaphragm is used in the deformation region of the blank. In this region, the liquid is in direct contact with the blank. Thus, while the diaphragm is prevented from any deformation and tearing, lower pressure is required to form the workpiece, compared with the case in the standard hydroforming.

In contrast to the conventional deep drawing, the sheet metal is not in direct contact with the die in the proposed method. Also, the blank holding system in this method is a soft-tool one. Therefore, wrinkles can be controlled to high extent, compared with the case in the hydromechanical and hydro-rim processes. 

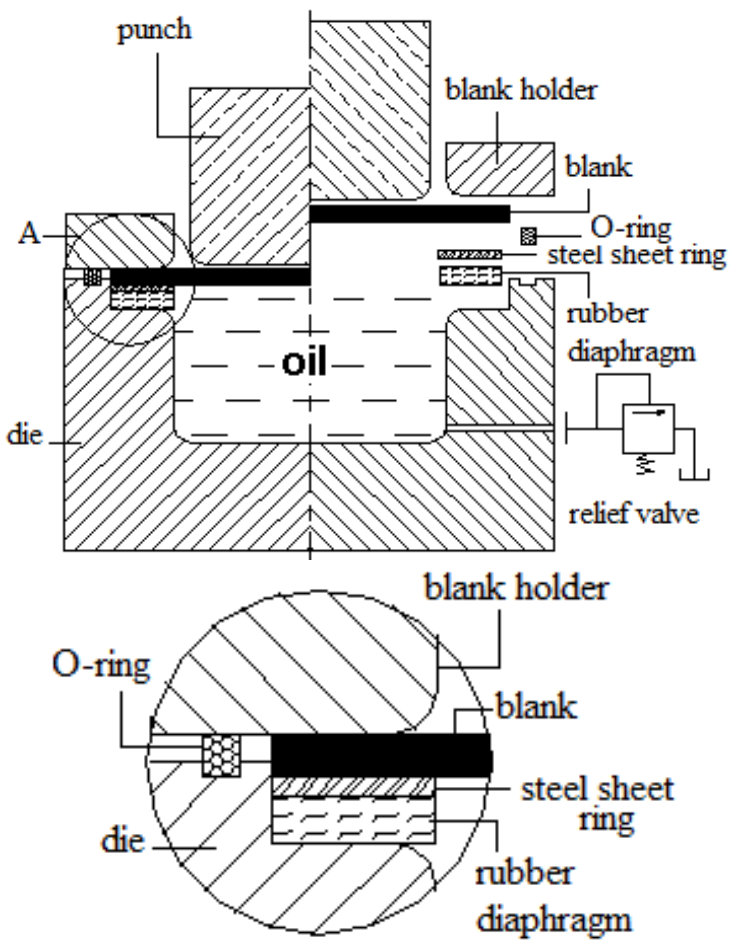

\section{detail A}

Figure 10. Schematic illustration of the tool set-up in the proposed method

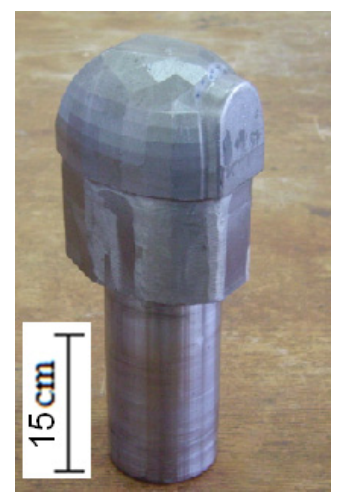

Figure 11. Photograph of the manufactured punch for case study I

The operational sequence for the new hydroforming process is given below:

1. The die was clamped on the machine anvil. The rubber diaphragm was located on the die, the steel sheet was put on the rubber, and the O-ring was located in the groove 
machined in the die. Then, the die cavity was filled with the pressure medium. The circular blank was then located on the steel sheet ring.

2. The blank was clamped between the die and blank holder by four screws. At this stage, an initial blank holder force was applied to the blank and a pre-hydroforming pressure was exerted on the lower surface of the rubber diaphragm and blank, lead to a small preloading on the blank.

3. The punch that was attached to the machine ram, moved down, pressurized the medium, and the deformation was started.

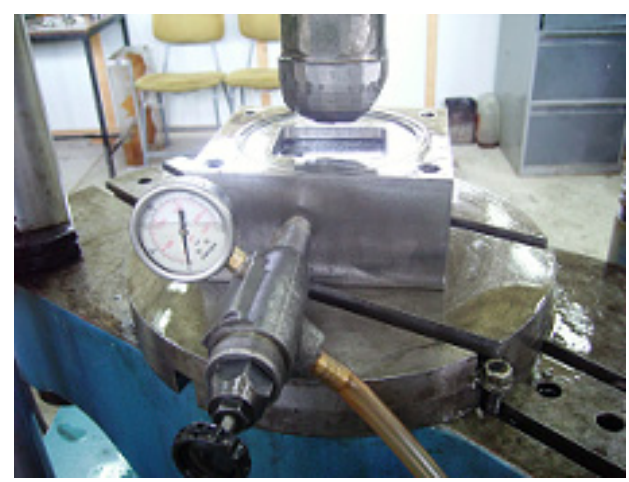

Figure 12. Photograph of the manufactured die set

Figure 13(a) illustrates the variations of internal pressure with punch stroke for the hydroforming of part in case study 1 . As it is seen from the figure, the maximum forming pressure is about $5.5 \mathrm{MPa}$ that is very low, compared with the results of reference [16], which formed simple parts with other hydroforming processes. As it can be seen from Figure 13(a), the internal pressure in the final stage of hydroforming oscillates. This is due to forming the special internal profile of the workpiece. Figure 13(b) illustrates the load-punch stroke curve of the workpiece. As it is seen from the figure, the maximum load is about $60 \mathrm{kN}$ which is not so high.

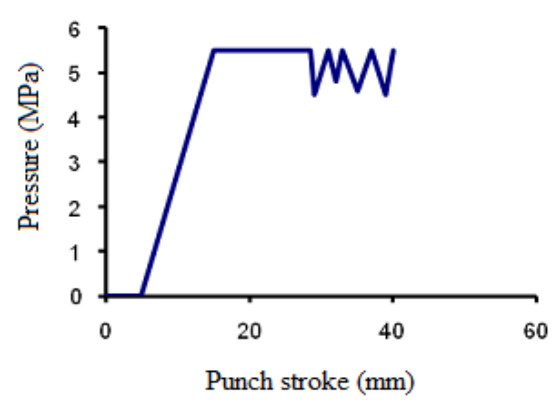

(a)

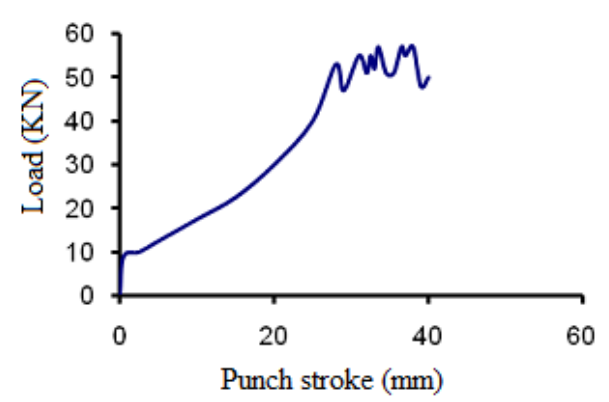

(b)

Figure 13. (a) Internal pressure-punch stroke curve, (b) load-displacement curve, correspond to case study I 


\section{Tool set-up: Case study II}

In this study, pure copper and St14 sheets with different thicknesses and different initial diameters were used to form conical-cylindrical cups in one stage by HDDRP process. Mechanical and physical properties for the sheets are shown in Table 1. Based on the experimental observations, the copper sheet did no behave any anisotropy. To characterize the material properties and anisotropy for steel sheets, according to ASTM-A370 standard different specimens were cut at different orientations to the rolling directions $\left(0^{\circ}, 45^{\circ}\right.$, and $90^{\circ}$ ). Tensile specimens were used to determine the stress-strain curves and the sheet anisotropy parameters, r-values. Plastic strain ratio (r-value) and yield stress ratio (R-value) for St14 sheet are illustrated in Table 2.

The schematic of the hydroforming die set used in this research is shown in Figure 14. Figure 15 shows the parametric geometry of the workpieces examined. Initially, three types of blanks with similar geometries but with variation in material or sheet thickness were selected. The specifications of these blanks and the formed parts are shown in Table 3 as parts $A, B$ and $C$ and with strain hardening equation of $\sigma=k \varepsilon^{n}$, where $k$ and $n$ are specified in the table.

A component with different geometry from those of the above mentioned parts was also selected to have a wider study. This is specified in Table 3 as part D.

\begin{tabular}{c|cccccc}
$\begin{array}{c}\text { Blank } \\
\text { material }\end{array}$ & $\begin{array}{c}\text { Young's } \\
\text { modulus, } \\
\mathrm{E}(\mathrm{GPa})[16]\end{array}$ & $\begin{array}{c}\text { Strain hardening } \\
\text { exponent, } \mathrm{n}\end{array}$ & $\begin{array}{c}\text { Strength } \\
\text { coefficient, K }\end{array}$ & $\begin{array}{c}\text { Yield stress, } \sigma \\
(\mathrm{MPa})[16]\end{array}$ & $\begin{array}{c}\text { Poisson's } \\
\text { ratio [16] }\end{array}$ & $\begin{array}{c}\text { Density, } \\
\rho\left(\mathrm{kg} / \mathrm{m}^{3}\right)\end{array}$ \\
{$\left[\begin{array}{c}16] \\
\text { Cu 99.9\% }\end{array}\right.$} & 117 & 0.44 & 530.98 & 123 & 0.32 & 8940 \\
St 14 & 210 & 0.35 & 638.96 & 190 & 0.3 & 7850 \\
\hline
\end{tabular}

Table 1. Mechanical and physical properties for pure copper and St14 sheets

\begin{tabular}{|c|c|c|c|c|c|c|}
\hline Thickness $(\mathrm{mm})$ & R0 & R45 & R90 & R22 & R33 & R12 \\
\hline 1 & 1.79 & 2.27 & 1.01 & 1.0402 & 1.24897 & 1.0789 \\
\hline
\end{tabular}

Table 2. Plastic strain ratio (r-value) and yield stress ratio (R-value) for St14 sheet

A DMG (Denison Mayes Group) universal testing machine with $600 \mathrm{kN}$ capacity was used in the experiments.

Figure 16 shows the components of the die and the assembled die-set. Figure 17 shows the parametric dimensions of the die set used for forming the parts. The dimensions of the die set are given in Table 4 . 


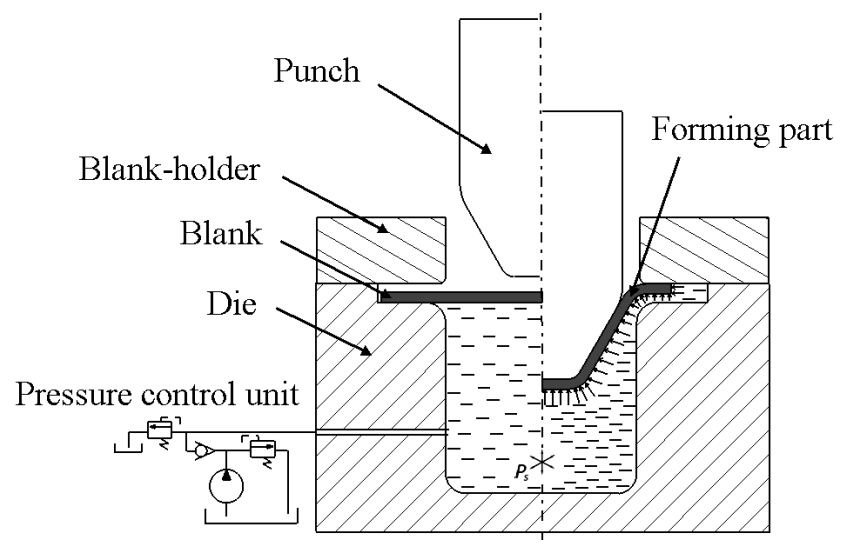

Figure 14. Schematic of the hydroforming die set used

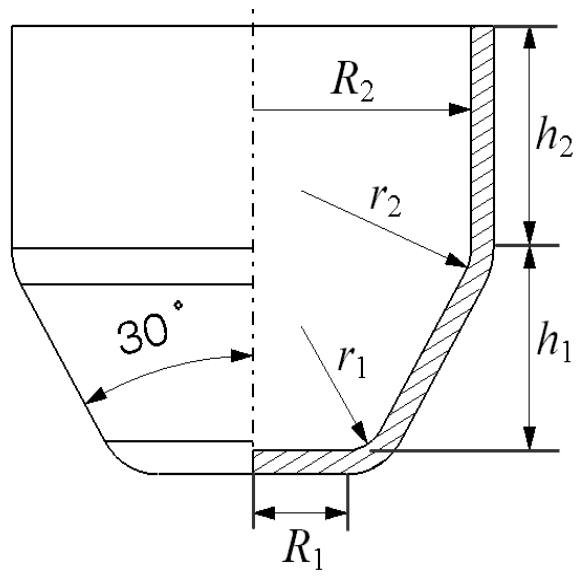

Figure 15. Parametric dimensions of formed parts

\begin{tabular}{l|c|c}
\multirow{2}{*}{ Type and dimensions of the part } & \multicolumn{2}{c}{ Values related to parts } \\
\cline { 2 - 3 } Type of material & Parts (A, B, C) & Part (D) \\
Height of conical portion, $\mathrm{h}_{1}$ & $\mathrm{~A}, \mathrm{~B}=$ Pure Copper, C=St14 & Pure copper \\
Height of cylindrical portion, h2 & 20 & 40 \\
Flat head radius, R1 & 18 & 16 \\
Cylindrical radius section, $\mathrm{R}_{2}$ & 8 & 7.5 \\
Nose radius, r1 & 20.75 & 35.35 \\
Conical-cylindrical radius, $\mathrm{r}_{2}$ & 3.5 & 10.5 \\
Initial blank thickness to & 5.5 & 6.5 \\
\hline
\end{tabular}

Table 3. Parameters of the part corresponds to Figure 15 (Dimensions in $\mathrm{mm}$ ) 

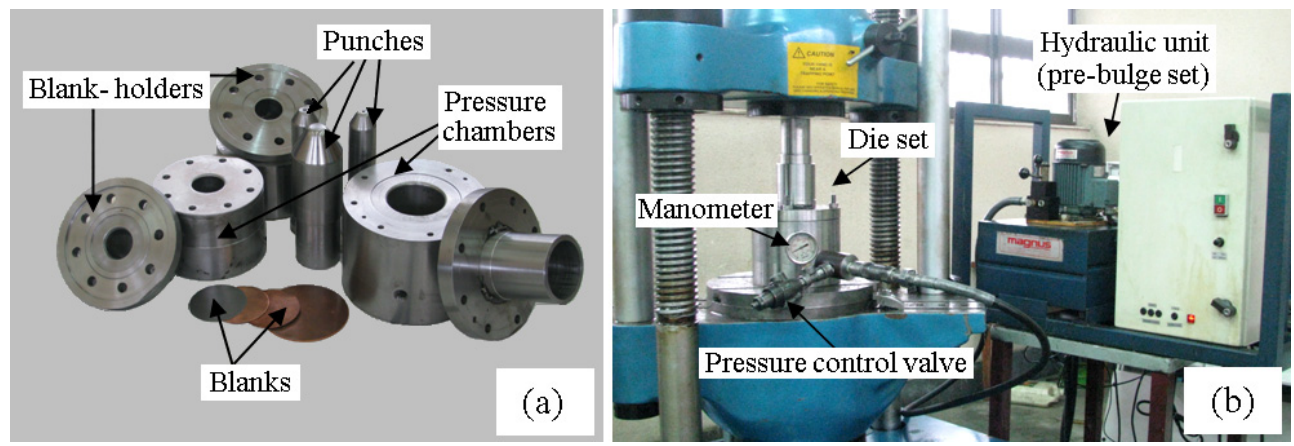

Figure 16. (a) components of the die, (b) assembled die set mounted on the test machine

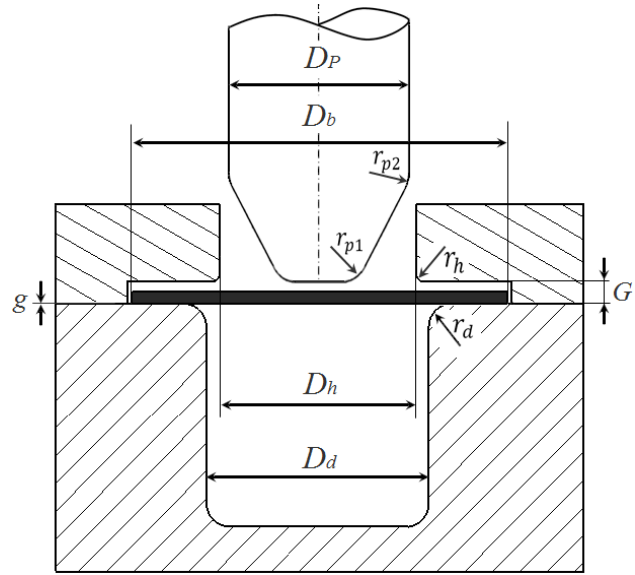

Figure 17. Parametric dimensions of the hydroforming die set

\begin{tabular}{l|cc|}
\multicolumn{1}{c}{ Parameter } & \multicolumn{2}{c|}{ Values related to parts } \\
\cline { 2 - 3 } \multicolumn{1}{c}{ Die inside diameter, Dd } & Parts A, B, C & Part D \\
diameter, Dh Blank holder inside & $\mathrm{A}=46, \mathrm{~B}=\mathrm{C}=44$ & 75 \\
Blank diameter, Db & 42 & 71 \\
Blank holder entrance radius, rh & 78 & 120 \\
Die entrance radius, rd & 3 & 3 \\
, G Gap between die and blank-holder & 4 & 5 \\
\hline
\end{tabular}

Table 4. Parameters of the die corresponds to Figure 17 (Dimensions in $\mathrm{mm}$ )

In the HDDRP, before the punch goes down, a small pre-bulging can be created on the sheet to improve the drawing process [16]. A hydraulic unit was used to create the pre-bulging pressure. When the punch moves down, the blank is forced into the die cavity filled with oil or other liquids. A control valve was used for controlling the maximum pressure. The liquid in the die cavity was pressurized so it pushes the blank tightly onto the punch surface. After 
reaching a maximum pressure, the control valve was opened and the pressure remained constant during the forming process. The liquid in the die cavity leaks out dynamically from the interface between the blank-holder and the die. The interface between die and blankholder was grinded metal contact and no o-ring was used in the die. At the same time, the liquid leaking out from this interface creates a pressure around the outside rim of the blank. Therefore, it is impossible to create high pre-bulging pressure in this die set. In this research, $2 \mathrm{MPa}$ pre-bulging pressure was applied.

Figure 18 shows the typical pressure path used in this study. In this path, OA is the initial pre-bulging pressure (2 $\mathrm{MPa}$ ) applied before the punch moves down. $\mathrm{BC}$ is the constant maximum pressure. The liquid outflows from control valve by applying this pressure. SAE10 hydraulic oil with a viscosity of $5.6 \mathrm{cSt}$ was used as the pressure medium. Due to the strain-rate sensitive behavior of the viscous medium, the punch velocity has significant effect on the internal pressure generation. Thus, in the pressure path of Figure 18, AB is the linear pressure path and its slope depends on punch velocity and workpiece shape and thickness. In this research, a punch velocity of $200 \mathrm{~mm} / \mathrm{min}$ was applied. To measure the cup thickness, a mechanical thickness measurement set was used.

The typical pressure path in this paper was shown in Figure 18. According to the figure, for each certain maximum pressure, a pre-bulging pressure, $\mathrm{OA}$, and a pressure path $\mathrm{AB}$ with different slopes are definable. The slope of $\mathrm{AB}$ changes with punch velocity, workpiece shape and sheet thickness. The punch velocity was fixed at $200 \mathrm{~mm} / \mathrm{min}$. Thus, for each certain part with defined shape and thickness, one specific slope was obtained.

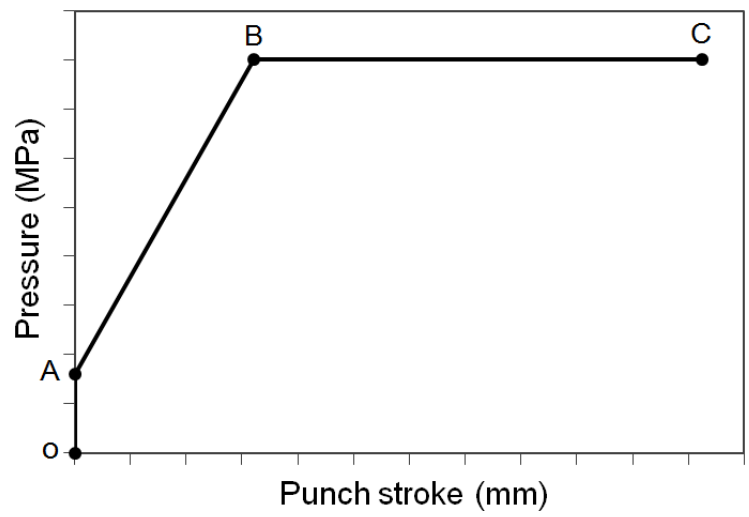

Figure 18. The typical pressure path applied in the investigation

\section{FEM simulation}

The commercial software, ABAQUS 6.7/Explicit, was used for the simulation. For pure copper sheet, the material behavior was assumed to be isotropic as the experimental results have verified this assumption. For St14 sheet, the anisotropy factors mentioned in the previous section were used in the simulation. 3D models were used for the simulation. The blank was modeled deformable with eight-node solid element (C3D8R). The number of 
elements along the thickness was 4 . The die set was modeled using a rigid four- node shell element (R3D4).

The die and the blank holder were constrained fully and the punch could move only along the vertical direction, corresponding to the central axis of the punch. Pressure constrains were applied on the whole bottom surface and also on the rim of the blank. The gap between the die and the blank holder was fixed. The punch motion was prescribed with a constant velocity. Because of the consideration of pre-bulging, the loading of liquid pressure in the die cavity was used as a two-step linear profile. The friction coefficient on the blank and the punch interface was considered to be 0.14 in the simulations. The coefficient on the other surfaces was considered to be 0.04 . Penalty contact interfaces were used between the sheet metal and the tooling elements. Table 1 shows the properties of pure copper and St14 sheets which have been used.

\section{Results and discussion}

\section{Case study I}

The schematic of the modified die-set for case study I is shown in Figure 19. The photograph of the used punch is shown in tool set-up section. To form this part, several pressure paths have been examined by FE simulation and the appropriate pressure path is shown in Figure 20. As it can be seen in the figure, the maximum forming pressure is about $5.5 \mathrm{MPa}$ which is very low, in comparison to the results of the other relevant references, which formed simple parts with other hydroforming processes.

Figure 21 shows the photograph of the workpiece formed in the new die-set. The initial blank is a round one with a diameter of $140 \mathrm{~mm}$. As it can be seen in this figure, the workpiece is formed quite well to the final required height, only in one step. The sharp region of the workpiece is formed successfully. The internal surface of the product is formed with high precision and good surface finish and there is no, even one small, wrinkle on the flange area.

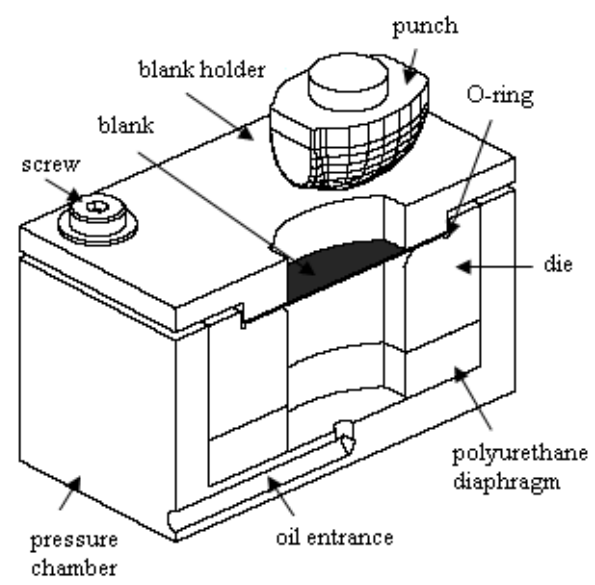

Figure 19. Schematic illustration of the proposed set-up for a complex part 


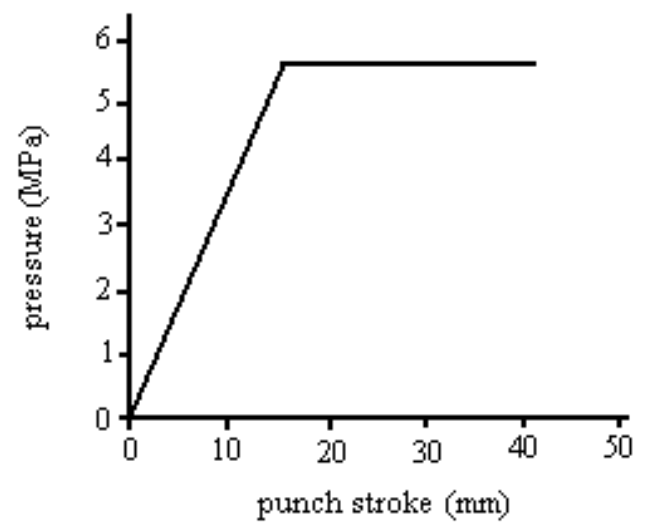

Figure 20. Pressure path used to form the lightning industry product

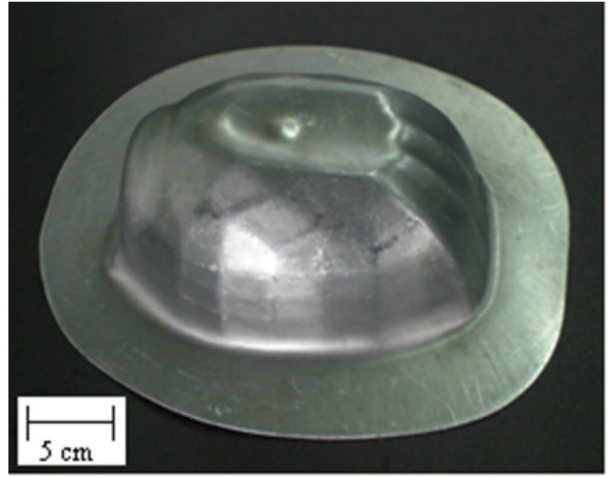

(a)

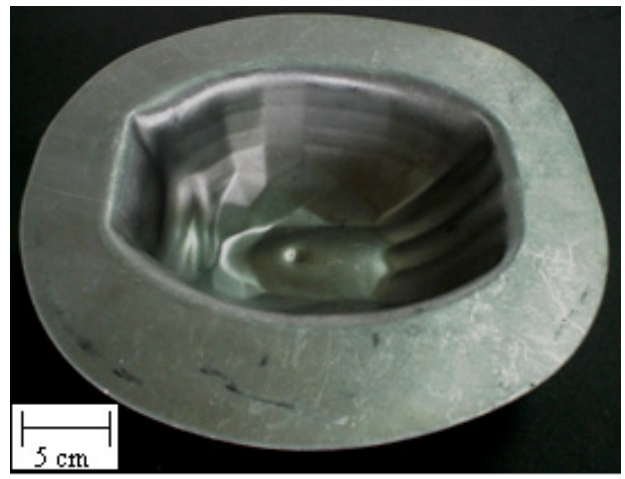

(b)

Figure 21. Photograph of the hydroformed part in the proposed die-set, (a) external view, (b) internal view

\section{Case study II}

Figure 22 shows the desired paths corresponding to maximum pressures for parts $\mathrm{A}, \mathrm{B}, \mathrm{C}$ and D. For parts B and C the punch velocity, workpiece shape and sheet thickness are the same which leads to the same slope. 


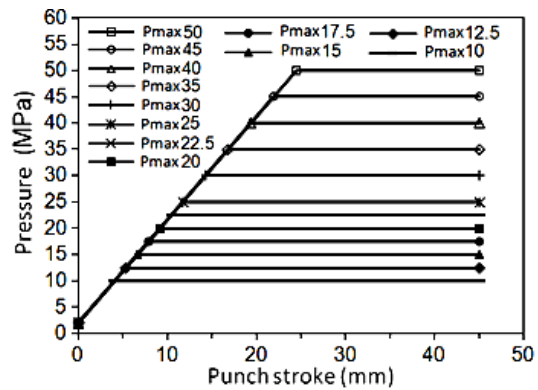

(a)

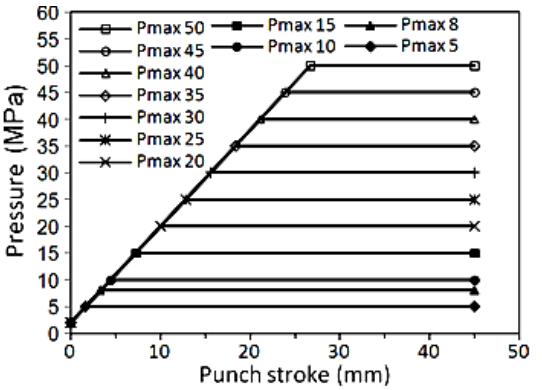

(b)

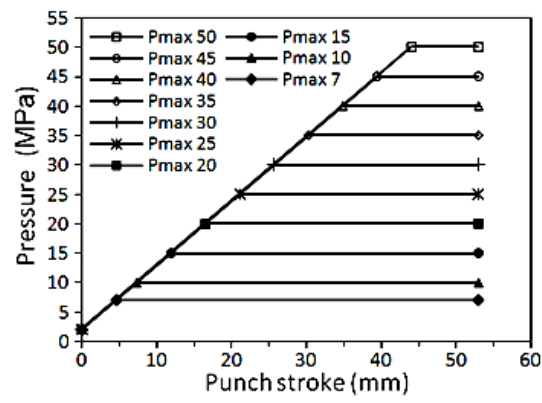

(c)

Figure 22. Desired pressure paths corresponding to different $P_{\max }$, to form (a) part A, (b) parts B, C, (c) part D

The results obtained from experiment and simulation illustrated that for part A with maximum pressures of less than $12.5 \mathrm{MPa}$ bursting occurs in the contact area of the workpiece with punch nose radius. Figure 23 shows a model of part A formed with maximum pressure of $10 \mathrm{MPa}$ along with its simulation results. As it can be seen, bursting has occurred in the workpiece because the low level of forming pressure leads this process to act just like as the conventional deep drawing.

The results indicated that in parts $\mathrm{B}, \mathrm{C}$, and D bursting occurs in the same area at maximum pressures less than 7.5, 17.5 and $7.5 \mathrm{MPa}$, respectively. Figure 24 shows the picture of parts $\mathrm{B}, \mathrm{C}$ and $\mathrm{D}$ in which bursting occurred.
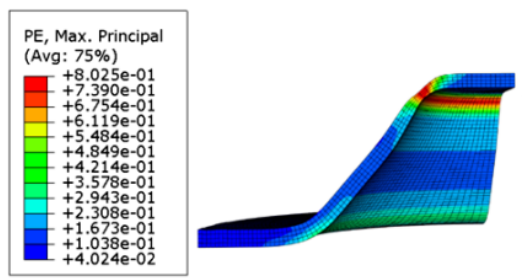

(a)

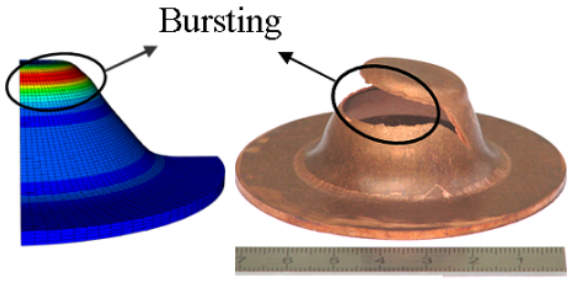

(b)

Figure 23. Deformed part A corresponds to pressure path $10 \mathrm{MPa}$, (a) simulation, (b) experiment 


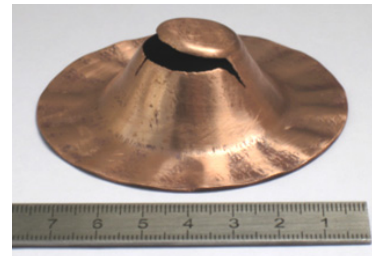

(a)

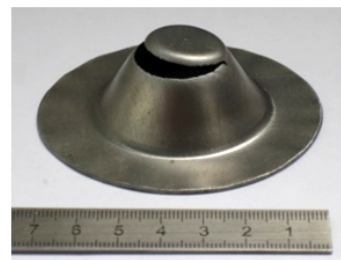

(b)

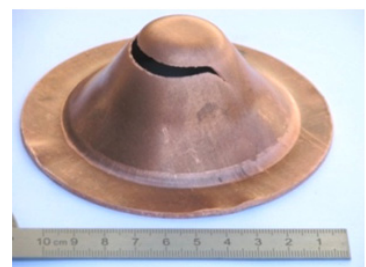

(c)

Figure 24. Deformed workpieces correspond to different pressure paths, (a) part $\mathrm{B}, \mathrm{P}_{\max }=5 \mathrm{MPa}$, (b) part C, $P_{\max }=15 \mathrm{MPa},(\mathrm{c})$ part D, $\mathrm{P}_{\max }=5 \mathrm{MPa}$

In part A with maximum pressure above $12.5 \mathrm{MPa}$ the conical cup was formed. Figure 25 shows the picture of part A formed with maximum pressure of $12.5 \mathrm{MPa}$. As it is seen, at the end of the process the conical cup was formed, but necking occurred in the workpiece.

The results indicated that in parts $\mathrm{B}, \mathrm{C}$, and D necking occurred in the same area at maximum pressures of 7.5, 17.5 and $7.5 \mathrm{MPa}$, respectively. Figure 26 indicates the picture of the parts in which necking occurred.
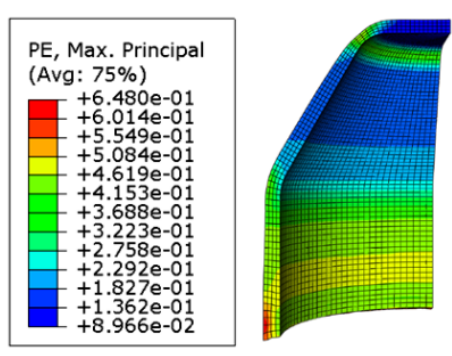

(a)

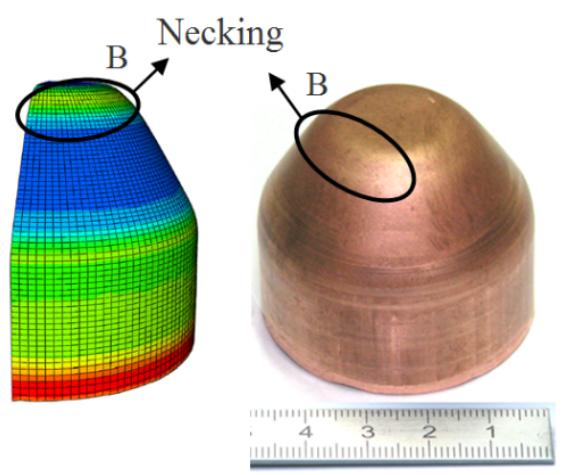

(b)

Figure 25. Deformed part A correspond to maximum pressure $12.5 \mathrm{MPa}$, (a) simulation, (b) experiment

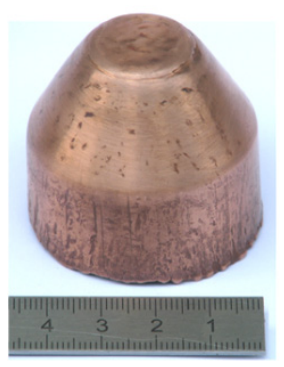

(a)

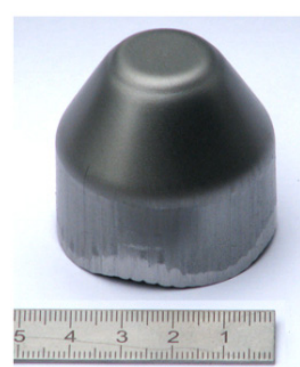

(b)

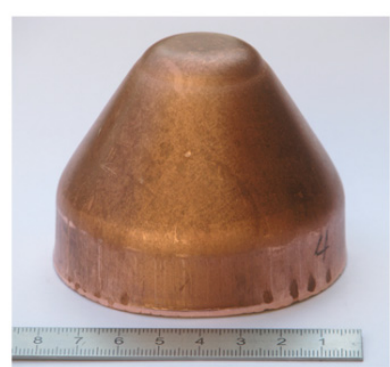

(c)

Figure 26. Deformed workpieces corresponds to pressure paths, (a) part $\mathrm{B}, \mathrm{P}_{\max }=7.5 \mathrm{MPa}$, (b) part $\mathrm{C}$, $\mathrm{P}_{\max }=17.5 \mathrm{MPa}$, (c) part $\mathrm{D}, \mathrm{P}_{\max }=7.5 \mathrm{MPa}$ 
It was observed that increasing the maximum pressure leads to decrease necking. Figure 27 indicates the formed part A corresponding to $25 \mathrm{MPa}$ maximum pressure. Applying this maximum pressure at final stage causes complete forming of the cup without necking defect and creates an accurate geometry.
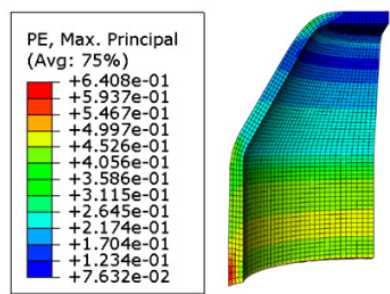

(a)

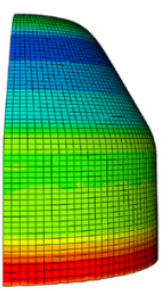

(a)

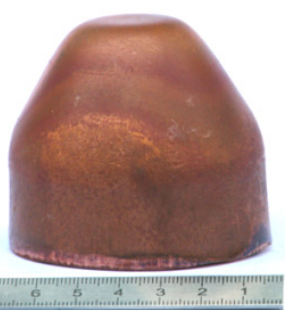

(b)

Figure 27. Deformed part A corresponds to pressure path $25 \mathrm{MPa}$, (a) simulation, (b) experiment

Parts B, C and D were formed without any necking occurrence with the maximum pressure paths of 17.5, 35 and $20 \mathrm{MPa}$, respectively, and are shown in Figure 28.

To have more careful study of thickness distribution, the deformed cups were divided into different regions as it is shown in Figure 29.

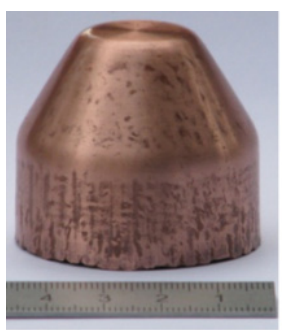

(a)

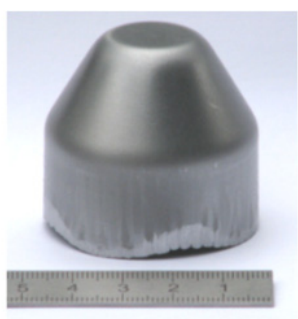

(b)

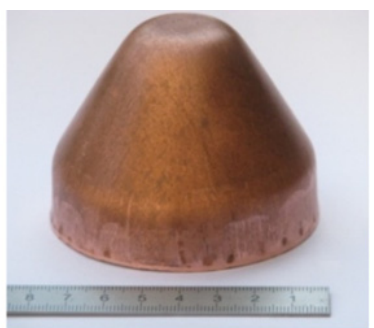

(c)

Figure 28. Deformed workpieces, (a) part $\mathrm{B}, \mathrm{P}_{\max }=17.5 \mathrm{MPa}$, (b) part $\mathrm{C}, \mathrm{P}_{\max }=35 \mathrm{MPa}$, (c) part $\mathrm{D}, \mathrm{P}_{\max }=$ $20 \mathrm{MPa}$

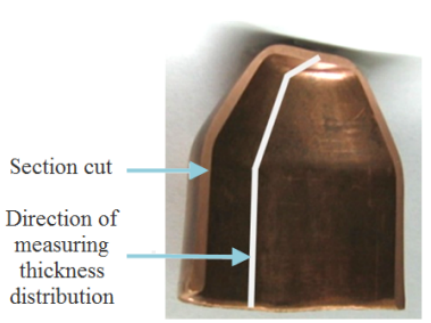

(a)

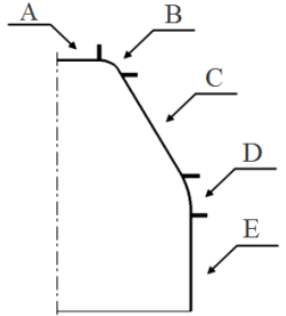

(b)

Figure 29. (a) Direction of measuring thickness distribution of conical parts, (b) different regions on the formed part 
Figure 30 shows the thickness distribution curve for part A with maximum pressure of 25 $\mathrm{MPa}$. As it is seen from the figure, there is a good correlation between the results of simulation and experiment.

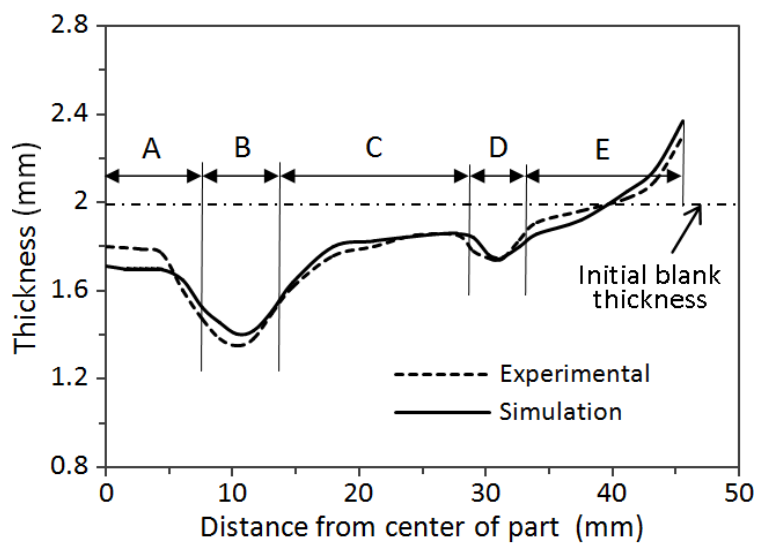

Figure 30. Thickness distribution curves for part A in maximum pressure of $25 \mathrm{MPa}$

In Figure 31 the thickness distribution curves obtained from experiment for different maximum pressures for four parts are displayed. As it can be found from this figure in the top of the conical cup, region A, the thickness reduction is very small. The most thickness reduction occurred in $\mathrm{B}$ and $\mathrm{D}$ regions. This thickness reduction is because of the bending occurrence in these regions. Region $\mathrm{B}$ is the critical zone as it was indicated in the previous results. In $\mathrm{C}$ and $\mathrm{E}$ regions the thickness increases and this thickness increase becomes greater toward the edge. It is obvious that the pressure increasing has a great effect on thickness reduction at different points of cup, especially in the critical region B.

For obtaining the best forming pressure path to produce a cup with better thickness distribution and quality, the maximum thickness reduction curve in B region was compared for different pressures. As stated previously, this is the most critical region of conical formed parts. Figure 32 shows the thickness distribution curves in B region corresponding to pressure paths with different maximum fluid pressures. As it can be found in Figure 32 (a), in part A the greatest thickness reduction is related to maximum pressure path $12.5 \mathrm{MPa}$. At this pressure, necking defect occurred in region B. It can be seen in the figure that by increasing the maximum pressure to $25 \mathrm{MPa}$, thickness reduction decreases with sharp slope. From the maximum pressure of $25 \mathrm{MPa}$ and greater, the slope will not change considerably. Thus, maximum pressure more than $25 \mathrm{MPa}$ does not have any positive effect on the cup thickness in region B. Also, in Figure 32 (b), (c), (d) it can be found that the similar behavior happened for other parts but the greatest thickness reduction and minimum thickness reduction are different. 


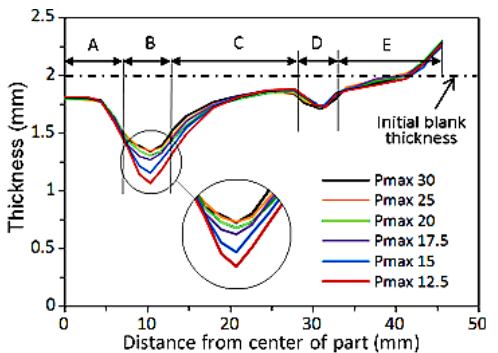

(a)

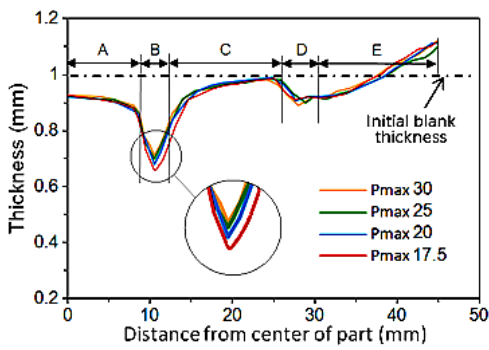

(c)

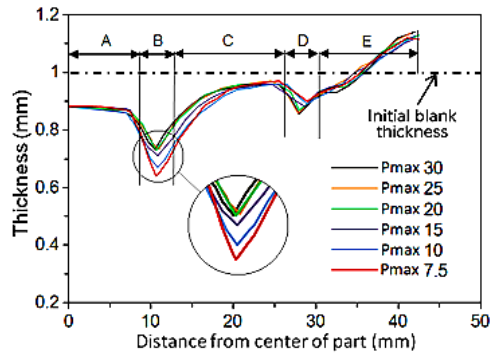

(b)

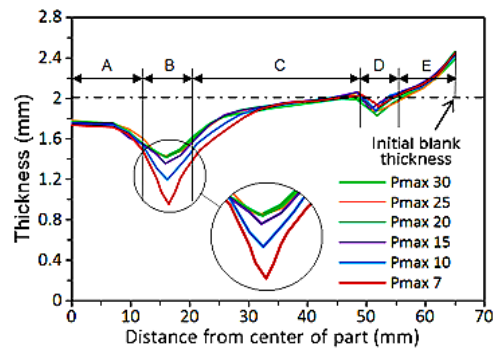

(d)

Figure 31. Thickness distribution curves for formed parts in different pressure paths, obtained from experiments, (a) part $\mathrm{A},(\mathrm{b})$ part $\mathrm{B},(\mathrm{c})$ part $\mathrm{C},(\mathrm{d})$ part $\mathrm{D}$

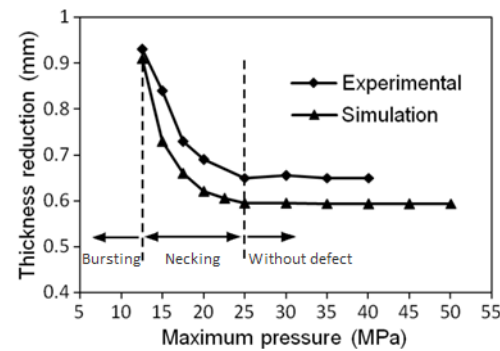

(a)

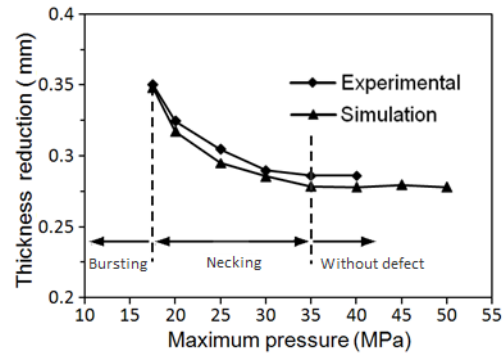

(c)

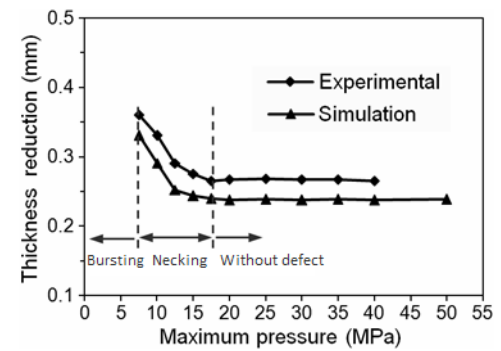

(b)

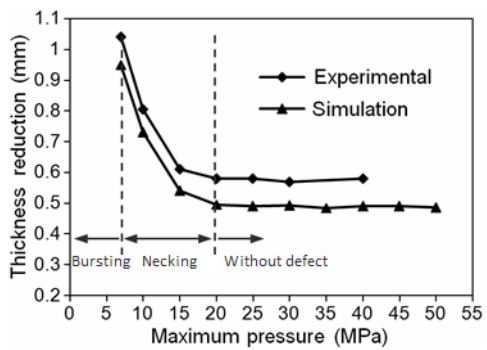

(d)

Figure 32. Thickness reduction curve versus maximum pressure for region $B$ of conical parts, (a) part A, (b) part B, (c) part C, (d) part D 
The drawing ratio of conical part is the ratio of initial blank diameter to the minimum diameter of the conical potion. The drawing ratio for parts $\mathrm{A}, \mathrm{B}$ and $\mathrm{C}$ is 4.875 and for part $\mathrm{D}$ is 8 . The relation between the drawing ratio and the lowest maximum forming pressure has been studied through simulation which is shown in Figure 33. In this figure it can be seen that with the sheet diameter decreasing, the conical part forming will be possible at lower pressures. When the sheet diameter or drawing ratio is increased, the forming pressure increases too, but with increasing the pressure, the sheet diameter increases to some extent. In the workpiece A, the sheet with the maximum of $87 \mathrm{~mm}$ in diameter can be formed through increasing the pressure but forming a blank with a diameter greater than $87 \mathrm{~mm}$ is not possible.

In Figure 33 the maximum drawing ratio for parts A, B, C and D are 5.4, 6.06, 5, and 9.67, respectively. In conical part forming through HDDRP, the sheet thickness, the conical angle, punch tip radius (B region) and sheet properties have great and considerable influence on the drawing ratio.

Punch force is related to the forming force and internal pressure in vertical direction. As it can be seen in Figure 34, the punch force increases when it moves down to reach a maximum force, and as the punch continues to move downward the punch force decreases.

By increasing the maximum pressure, the more punch force is needed. This is because the vertical direction force increases. Figure 35 illustrates the effect of maximum fluid pressure on punch force for parts A, B, C and D.

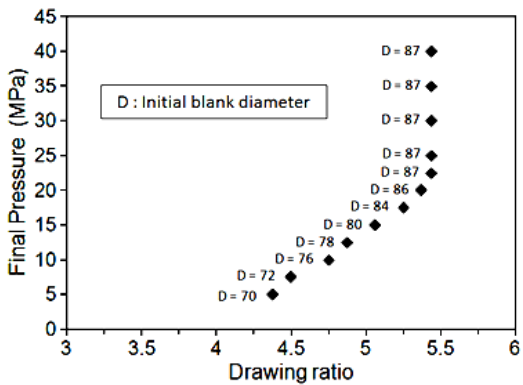

(a)

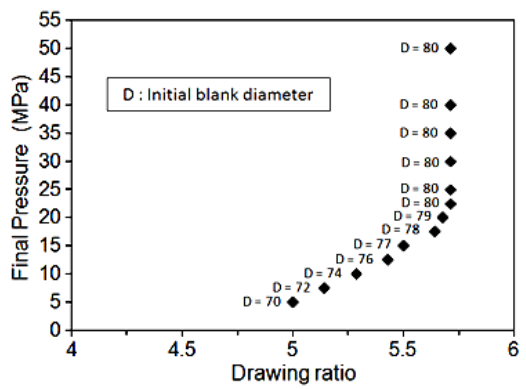

(c)

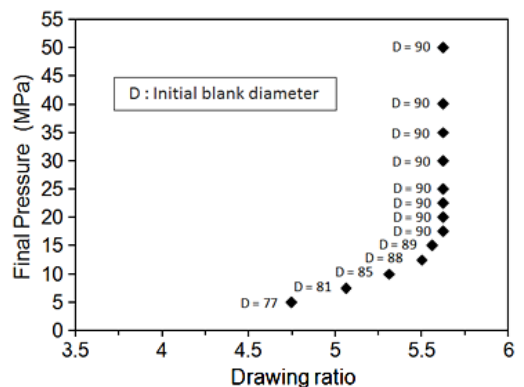

(b)

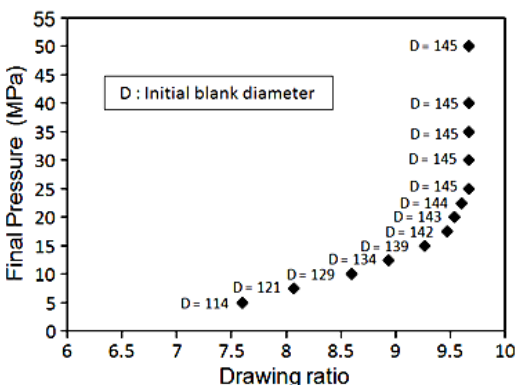

(d)

Figure 33. The drawing ratio corresponding to maximum pressure, $P_{\max }$ (a) part $A,(b)$ part $B$, (c) Part C, (d) part D 


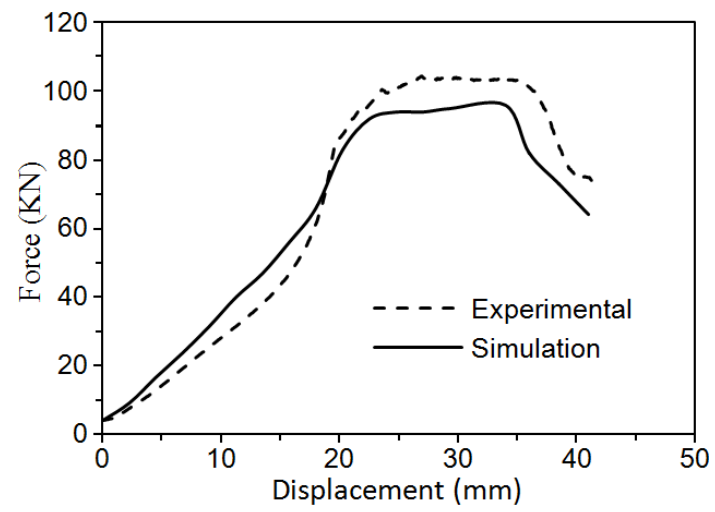

Figure 34. Force-punch stroke curve

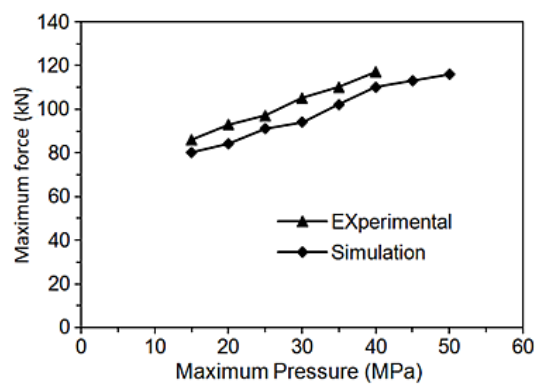

(a)

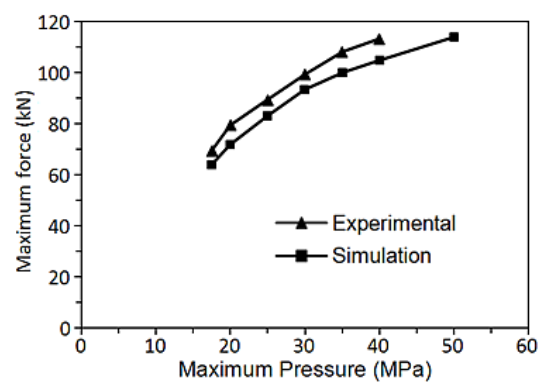

(c)

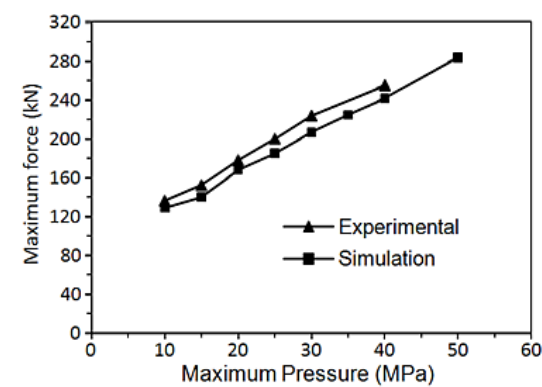

(b)

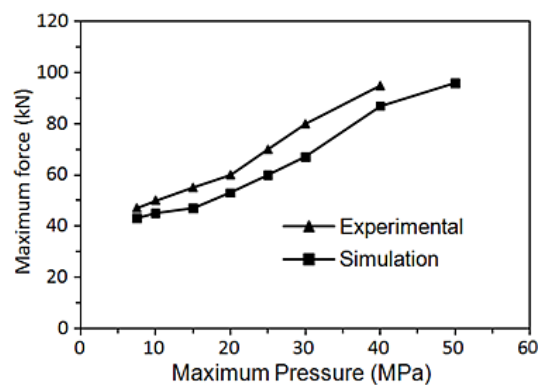

(d)

Figure 35. Maximum punch force versus maximum pressure corresponding to (a) Part A, (b) Part B, (c) Part C, (d) Part D

As it was stated, a pressure increase leads to a thickness reduction and on the other hand pressure increase makes the punch force more and there is a need to have bigger tonnage of press. So, obtaining the optimum pressure and punch force has a great importance in forming a workpiece with high quality.

To examine the effect of cone angle, different punches with $45^{\circ}, 60^{\circ}$ and $75^{\circ}$ angles were manufactured for part D geometry. Figure 36 shows \%thinning in B region corresponding to 
different pressure paths for conical part with different cone angles. As it can be found, in conical part with $45^{0}$ angle, the greatest thickness reduction is related to maximum pressure $18 \mathrm{MPa}$. At maximum pressure less than $18 \mathrm{MPa}$ bursting occurs in $\mathrm{B}$ region. It can be seen in the figure that by increasing the maximum pressure to $25 \mathrm{MPa}$, thickness reduction decreases with sharp slope. From the maximum pressure of $25 \mathrm{MPa}$ and greater, the slope will not change considerably. In conical workpiece with $75^{\circ}$ angle, without applying any pressure, no bursting was observed. With increasing the pressure to almost $20 \mathrm{MPa}$ the thickness reduction decreases sharply. At maximum pressure of approximately $20 \mathrm{MPa}$ and beyond this, the slope will be horizontal. Figure 36 shows \%thinning for the three different angles. It can be observed that with increasing the conical angle the thickness reduction will be decreased in B area. Moreover, as the conical angle increases, bursting occurs at lower pressure in such a way that beyond one specific angle, say $75^{\circ}$, the conical workpiece can be formed in the die chamber without applying any pressure.

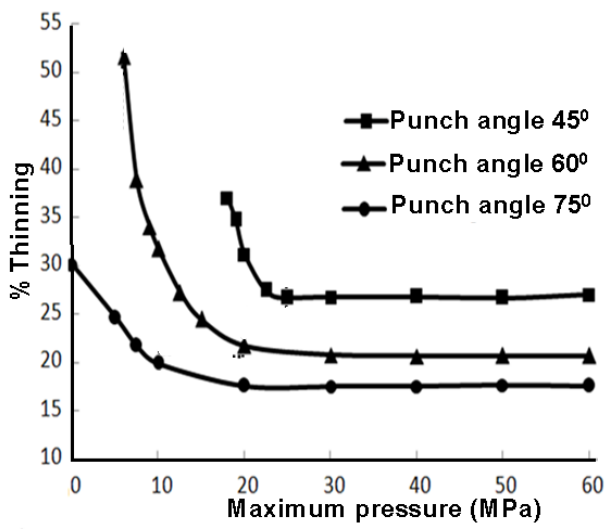

Figure 36. \% thinning for different cone angles

Figure 37 shows the failure, thinning and safe forming regions for different conical angles. As shown in the figure, by increasing the punch conical angle, the bursting region becomes smaller. At angle of $75^{\circ}$ and higher the part did not fail even without applying the fluid pressure. In other words, by decreasing the conical angle, the pressure level should increase to prevent the failure. This analysis is valid for safe forming limits too. It means that by increasing the angle, the maximum pressure level decreases for forming an accurate part without any defect. At the angle of less than $35^{\circ}$, bursting occurs in the part at any fluid pressure.

Figure 38 shows the effect of punch friction coefficient on thickness distribution of the conical part. As it is shown in the figure, changes in the punch friction coefficient only affect region $\mathrm{A}$ and $\mathrm{B}$, and it has no significant effect on other areas. It was observed that with increasing the punch tip radius the thickness reduction decreases in this region.

For more detailed review, the effect of punch friction coefficient in region A and B were studied for different punch friction coefficients. 


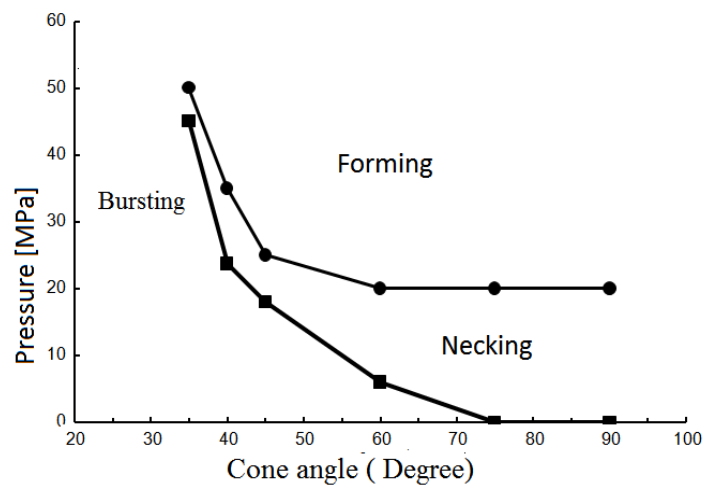

Figure 37. \% thinning versus cone angle

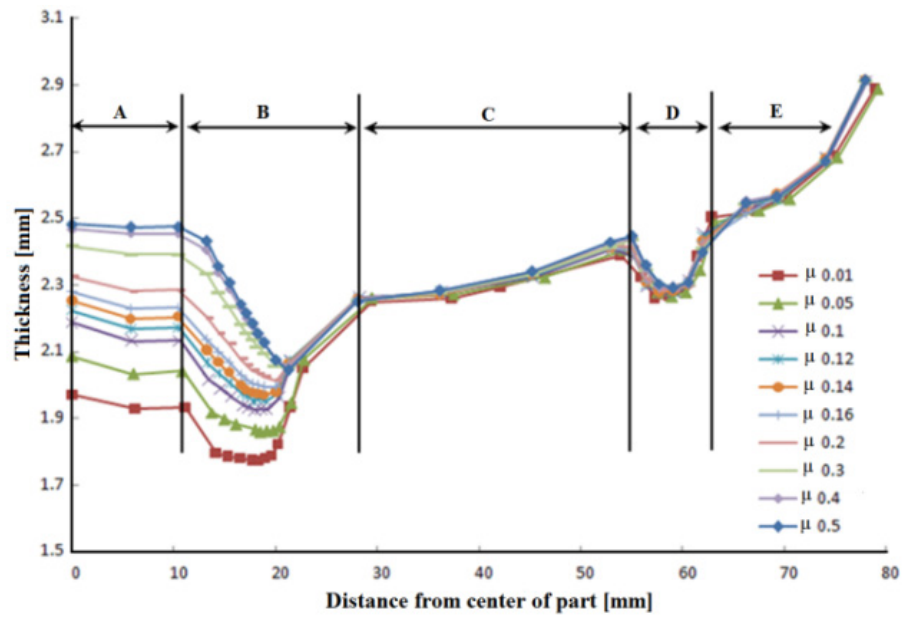

Figure 38. Thickness distribution curves versus punch friction coefficient, punch angle $60^{\circ}$

Figure 39 shows \%thinning curves in A and B regions corresponding to different punch friction coefficients. From the figure it is observed that as the punch friction coefficient increases the thickness reduction decreases in the two regions. It can be seen in the figure that by increasing the punch friction coefficient to 0.3 , thickness reduction decreases with sharp slope. From the punch friction coefficient greater than 0.3 the slope will not change considerably specially in B region.

Figure 40 illustrates the effect of the blank holder friction coefficient. As it shows, changes in the blank holder friction coefficient affect all regions. For more accurate study, the thickness reduction in B area was investigated and the results are shown in Figure 41. It is observed that by increasing the blank holder friction coefficient, the thickness reduction increases. At higher blank holder friction coefficients, necking occurs in B region. In this research, the blank holder friction coefficient of more than 0.3 results in bursting in region $\mathrm{B}$. 
Figure 42 shows the effect of sheet thickness on the thickness reduction in region B. As it is obvious, up to the maximum pressure of $20 \mathrm{MPa}$ the changes in the sheet thickness affect the thickness reduction. From the maximum pressure of $20 \mathrm{MPa}$ this effect is not considerable. Also, it can be observed that by decreasing the sheet thickness the possibility of thickness reduction reduces.

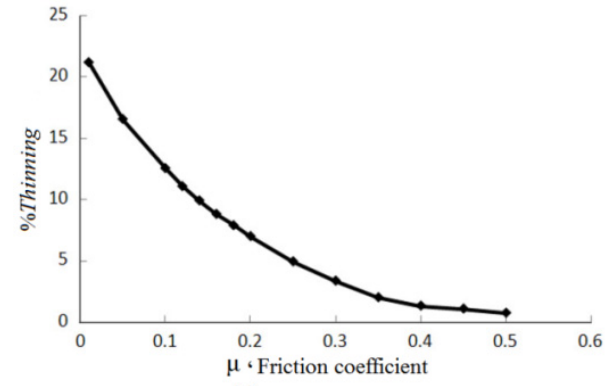

(a)

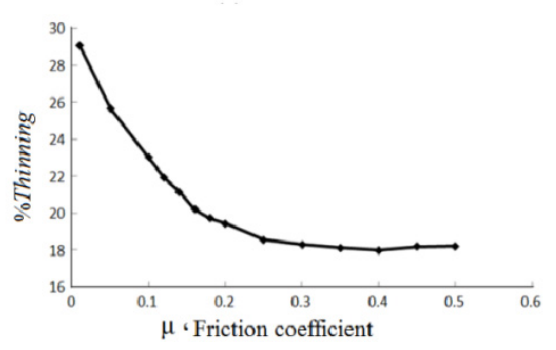

(b)

Figure 39. \% thinning of the conical part for different punch friction coefficient, (a) region A, (b) region B.

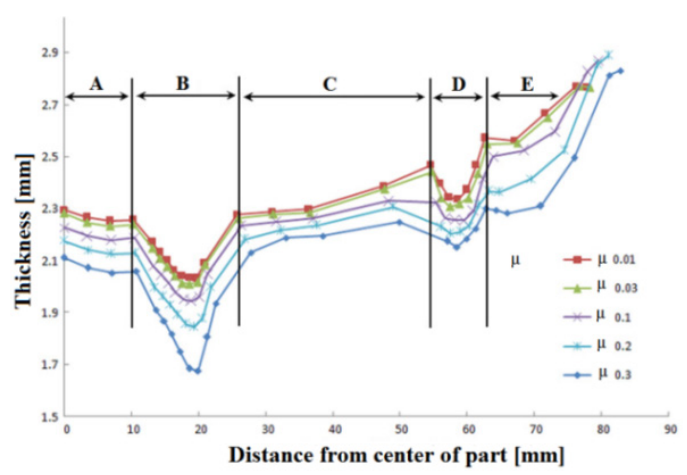

Figure 40. Thickness distribution curves for different blank holder friction coefficient, punch angle $60^{\circ}$

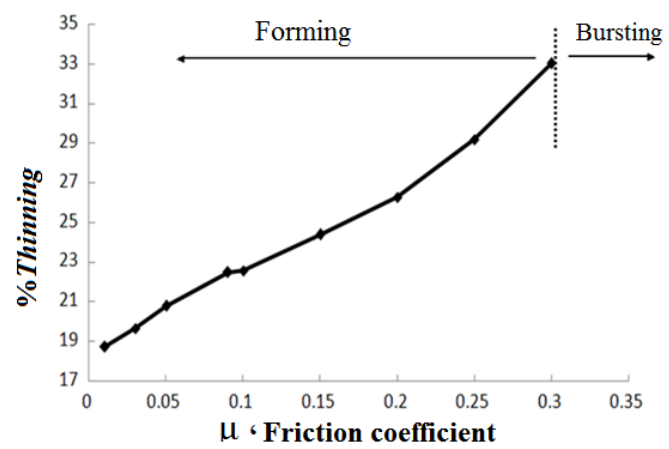

Figure 41. \% thinning versus blank holder friction coefficient in region $B$ 


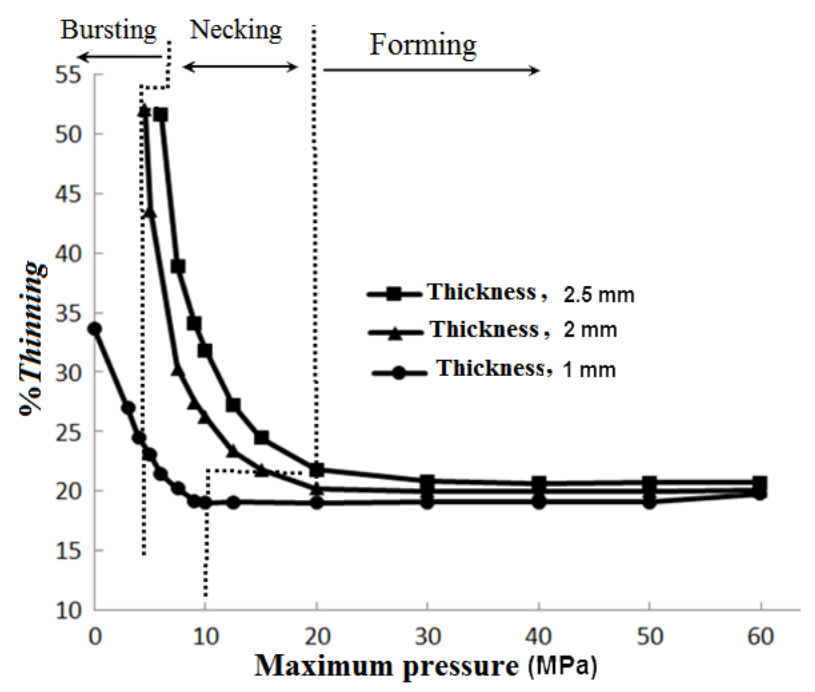

Figure 42. \% thinning versus sheet thickness

Bending radius is a very effective factor on thickness distribution. Figure 43 shows the effect of punch tip radius on thickness distribution of the conical part. As it is shown in the Figure, changing the punch tip radius only affects region $B$ and it has no significant effect on other areas.

Figure 44 shows \%Thinning at different punch radiuses. As it can be observed, with increasing the punch tip radius the thickness reduction decreases in B region. With increasing the conical angle the thickness reduction will be decreased in region B. Moreover, as the conical angle increases, bursting occurs at lower punch radius in such a way that beyond one specific degree angle, say $75^{\circ}$, the conical workpiece can be formed in the die chamber without applying any punch radius.

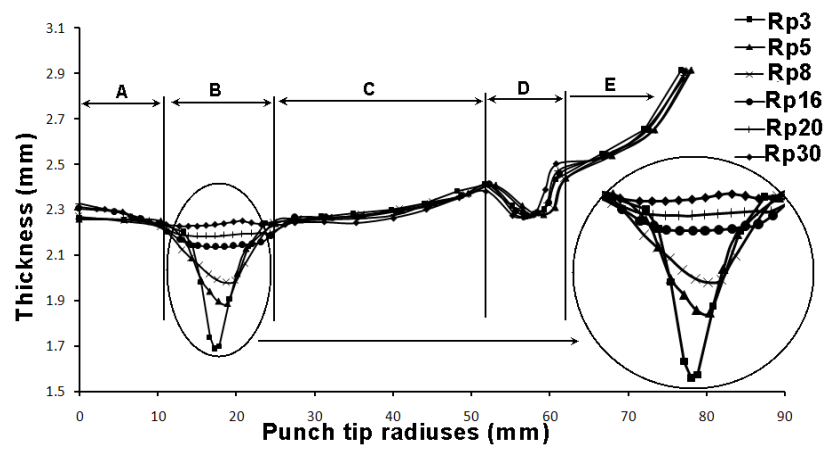

Figure 43. Thickness distribution curves versus punch tip radius, punch angle $60^{\circ}$ 


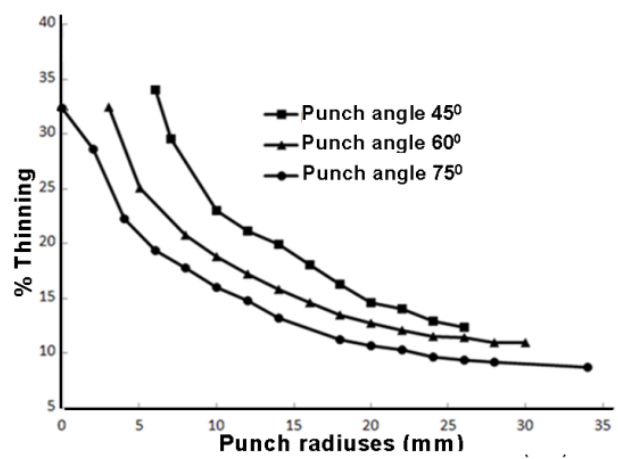

Figure 44. \% thinning versus punch tip radius for region $B$

Figure 45 illustrates the effect of the radius of conical-cylindrical region on the thickness distribution. As it shows, changes in the radius only affects region D and it has no effect on other regions of the part. For more accurate study, the thickness reduction in region $\mathrm{D}$ was investigated and the results are shown in Figure 46. As it can be seen, by increasing the radius of region $\mathrm{D}$ and the conical angle the thickness reduction will be decreased.

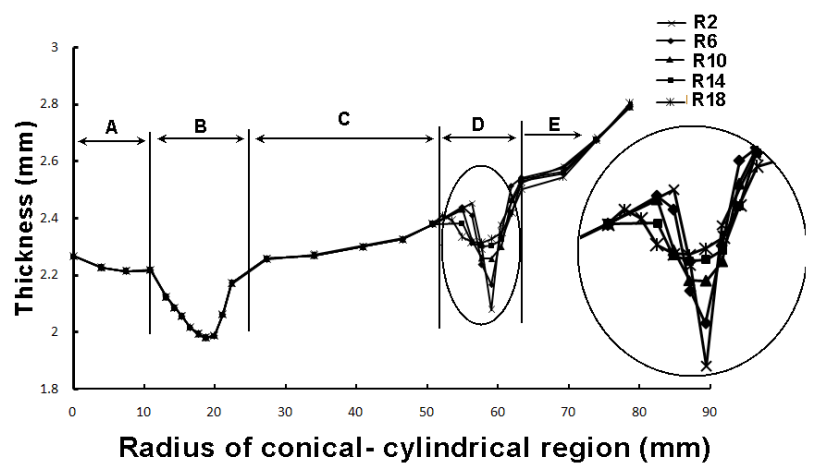

Figure 45. Thickness distribution curve versus radius of conical-cylindrical region

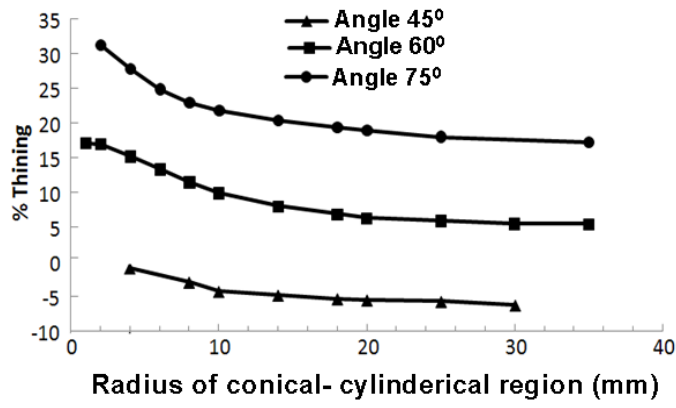

Figure 46. \% thinning versus radius of conical-cylindrical region 


\section{Conclusions}

In this chapter, a new sheet hydroforming is proposed and applied for forming of two industrial parts that currently are produced in industry by conventional deep drawing and stamping in several stages. With the new method, these two parts were produced in one stage and without any defects. In addition, it is shown that the forming pressure and load are very low compared with those of other hydroforming methods.

In addition, for case study II, the effects of tool parameters such as the radius of the punch tip, punch-cylindrical radius, friction between punch and sheet, friction coefficient between blank holder and sheet, sheet thickness and punch angle, on formability and thickness distribution of the conical parts were studied through using hydrodynamic deep drawing assisted by radial pressure. It was observed that with increasing the conical workpiece angle the thickness reduction will decrease in B area. Moreover, as the conical angle increases, bursting occurs at lower pressure in such a way that beyond one specific angle, the conical workpiece can be formed in the die chamber without applying any pressure. Also, with the cone angle increasing, the thickness distribution will be improved and the likelihood of bursting decreases.

\section{Author details}

M. Bakhshi-Jooybari, A. Gorji and M. Elyasi

Faculty of Mechanical Engineering, Babol University of Technology, Babol, Mazandaran, Iran

\section{Acknowledgement}

The authors would like to thank Dr. M. Hosseinzade for the provision of valuable information and product data.

\section{References}

[1] Singh H (2003) Fundamental of hydroforming, SME, 219 p.

[2] Koc M (2008) Hydroforming for advanced manufacturing, Woodhead publishing limited, $396 \mathrm{p}$.

[3] Elyasi M, Bakhshi-Jooybari M, Gorji A (2009) Mechanism of improvement of die corner filling in a new hydroforming die for stepped tubes. Materials and Design. 30: 38243830.

[4] Elyasi M, Bakhshi-Jooybari M, Gorji A, Hossinipour SJ, Norouzi S (2009) New die design for improvement of die corner filling in hydroforming of cylindrical stepped tubes. Proc. IMechE, Part B: J. Engineering Manufacture. 223: 821-827.

[5] Bakhshi-Jooybari M, Elyasi M, Gorji A (2009) Numerical and experimental investigation of the effect of the pressure path on forming metallic bellows. Proc. IMechE, Part B: J. Engineering Manufacture. 224: 95-101. 
[6] Elyasi M, Bakhshi-Jooybari M, Gorji A, Hossinipour SJ, Norouzi S (2008) Numerical and experimental investigation on forming metallic bellows in closed and open die hydroforming. Steel Research International. 79: 148-154.

[7] Khanlari H, Elyasi M, Bakhshi-Jooybari M, Gorji A, Davoodi B, Mohammad Alinegad G (2010) Investigation of Pressure Path Effect on Thickness Distribution of Product in Hydroforming Process of SS316L Seamless Tubes. Steel research international. 81: 560563.

[8] Elyasi M, Zoghipour P, Bakhshi-Jooybari M, Gorji A, Hosseinipour SJ, Nourouzi S (2010) A New Hydroforming Die Design for Improvement of Die Corner Filling of Conical Stepped Tubes. Steel research international. 81: 516-519.

[9] Hosseinzade M, Mostajeran H, Bakhshi-Jooybari M, Gorji A, Norouzi S, Hossinipour SJ (2009) Novel combined standard hydromechanical sheet hydroforming process, IMechE Journal of Engineering Manufacture, 224: 447-457.

[10] Thiruvarudchelvan S, Lewis W (1999) A note on hydroforming with constant fluid pressure. J. Mater. Process. Technol., 88: 51-56.

[11] Kandil A (2003) An experimental study of hydroforming deep drawing. J. Mater. Process. Technol. 134: 70-80.

[12] Zhang SH, Danckert J (1998) Development of hydro-mechanical deep drawing. J. Mater. Process. Technol. 83: $14-25$.

[13] Tirosh J, Shirizly A, Ben-David D, Stanger S (2000) Hydro-rim deep-drawing processes of hardening and rate-sensitive materials. Int. J. Mech. Sci. 42: 1049-1067.

[14] Groche P, Metz C (2006) Investigation of active-elastic blank holder systems for highpressure forming of metal sheets. Int. J. Mach. Tools Manufact. 46: 1271-1275.

[15] Groche P, Metz C (2005) Hydroforming of unwelded metal sheet using active-elastic tools. J. Mater. Proc. Tech. 168: 195-201

[16] Lang L, Danckert J, Nielsen KB (2004) Investigation into hydrodynamic deep drawing assisted by radial pressure Part I. Experimental observations of the forming process of aluminum alloy. J Mater Process Technol 148:119-131.

[17] Gorji A, Alavi-Hashemi A, Bakhshi-Jooybari M, Norouzi S, Hossinipour SJ (2011) Investigation of hydrodynamic deep drawing for conical-cylindrical cups. Int. J. of Advanced Manufacturing Technology. 56: 915-927.

[18] Lange K, (1985) Handbook of Metal Forming. McGraw-Hill Book Company, New York.

[19] Kawka M, Olejnik L, Rosochowski A, Sunaga H, Makinouchi A (2001) Simulation of wrinkling in sheet metal forming. J Mater Process Technol 109:283-289.

[20] Wong CC, Dean TA, Lin J (2003) A review of spinning, shear forming and flow forming processes. Int J Mach Tools Manufact 43:1419-1435.

[21] Liaghat G.H, Darvizeh A, Javabvar D, Abdollah A (2002) Analysis of explosive forming of conical cups, comparison of experimental and FEM simulation results. Amirkabir Journal 50:250-264 (in Persian). 